\title{
Frontal Lobe Anaplastic Astrocytoma
}

National Cancer Institute

\section{Source}

National Cancer Institute. Frontal Lobe Anaplastic Astrocytoma. NCI Thesaurus. Code C156035.

An anaplastic astrocytoma occurring in the frontal lobe. 